\title{
Anesthesia for minimally invasive cardiac surgery
}

\author{
Alexander White ${ }^{1}$, Chinmay Patvardhan ${ }^{2}$, Florian Falter ${ }^{2}$ \\ ${ }^{1}$ Senior Fellow in Anaesthetics and Intensive Care, ${ }^{2}$ Consultant in Anaesthetics and Intensive Care, The Royal Papworth Hospital NHS Foundation \\ Trust, Cambridge, UK \\ Contributions: (I) Conception and design: F Falter; (II) Administrative support: None; (III) Provision of study materials or patients: None; (IV) \\ Collection and assembly of data: None; (V) Data analysis and interpretation: None; (VI) Manuscript writing: All authors; (VII) Final approval of \\ manuscript: All authors. \\ Correspondence to: Florian Falter. Consultant in Anaesthetics and Intensive Care, The Royal Papworth Hospital NHS Foundation Trust, Cambridge, \\ UK. Email: florian.falter@gmail.com.
}

\begin{abstract}
Due to its potential benefits and increased patient satisfaction minimal invasive cardiac surgery (MICS) is rapidly gaining in popularity. These procedures are not without challenges and require careful planning, pre-operative patient assessment and excellent intraoperative communication. Assessment of patient suitability for MICS by a multi-disciplinary team during pre-operative workup is desirable. MICS requires additional skills that many might not consider to be part of the standard cardiac anesthetic toolkit. Anesthetists involved in MICS need not only be highly skilled in performing transesophageal echocardiography (TEE) but need to be proficient in multimodal analgesia, including locoregional or neuroaxial techniques. MICS procedures tend to cause more postoperative pain than standard median sternotomies do, and patients need analgesic management more in keeping with thoracic operations. Ultrasound guided peripheral regional anesthesia techniques like serratus anterior block can offer an advantage over neuroaxial techniques in patients on anti-platelet therapy or anticoagulation with low molecular weight or unfractionated heparin The article reviews the salient points pertaining to pre-operative assessment and suitability, intraoperative process and postoperative management of minimally invasive cardiac procedures in the operating theatre as well as the catheterization lab. Special emphasis is given to anesthetic management and analgesia techniques.
\end{abstract}

Keywords: Minimal invasive cardiac surgery (MICS); anesthesia; regional anesthesia; transesophageal echocardiography (TEE)

Submitted Apr 29, 2020. Accepted for publication Aug 17, 2020.

doi: $10.21037 /$ jtd-20-1804

View this article at: http://dx.doi.org/10.21037/jtd-20-1804

\section{Introduction}

After the introduction of cardiopulmonary bypass (CPB) into clinical practice in the 1950 s cardiac surgery is now performed safely and to a high standard around the world. While cardiac operations are traditionally performed via a midline sternotomy, technological advancement, improved surgical and anesthetic expertise as well as patient expectations have led to an increased demand for minimally invasive cardiac surgery (MICS). Although this term is somewhat subjective, the American Heart Association defines MICS as cardiac surgery performed through a small chest wall incision that does not include a full sternotomy (1).

The scope of MICS is broad and includes surgery for coronary revascularization, valve repair and replacement, removal of atrial masses, repair of atrial septal defects (ASDs), and atrial fibrillation (AF) ablation procedures. Some are performed on $\mathrm{CPB}$ while others are performed on a beating heart. Transcatheter interventions have become a mainstay in many institutions, and the scope of these procedures is expanding with the growth of technology and expertise.

MICS procedures present a host of considerations for the anesthesiologist. Many of these depend on institutional 
practices, as well as the experiences and preferences of the teams involved. This article reviews the most common anesthetic preoperative, intraoperative and postoperative challenges in facilitating MICS procedures in the operating theatre and in the catheterization laboratory.

\section{Principles of MICS}

The surgical approach varies depending on the procedure. The majority require a thoracotomy, with or without single-lung ventilation (SLV) and may involve positions other than strictly supine. This surgical access may require a more tailored analgesic technique in order to minimize postoperative pain. Without a sternotomy, direct visualization of the heart and the great vessels may not be possible. The use of transesophageal echocardiography (TEE) is therefore necessary to provide surgical and hemodynamic guidance throughout most MICS procedures.

Arterial and venous cannulation are commonly performed via the femoral vessels, and cannula position is confirmed with TEE. Aortic cross clamping and myocardial protection, if necessary, can be achieved under direct vision with a special transthoracic clamping device followed by direct injection of cardioplegia into the aortic root or by inserting an endovascular balloon-tipped catheter (endoballoon) via the femoral or subclavian artery into the proximal ascending aorta and delivering cardioplegia through the distal tip of the catheter into the coronary ostia. Should retrograde cardioplegia be necessary, a coronary sinus catheter may be inserted, although the small surgical field can make this technically difficult and it is not frequently used.

Hemodynamic management and weaning from CPB follows the same principles as conventional cardiac surgery.

Postoperatively patients are managed in a similar fashion to traditional cardiac surgical patients. They are typically extubated within a few hours and their ICU length of stay is 24-48 hours (2). A multimodal approach to analgesia with both pharmacologic and non-pharmacologic methods is favored.

\section{Preoperative evaluation}

Similar to conventional cardiac surgery, the preoperative evaluation involves standard history, physical exam, laboratory investigations and imaging. However, there are several key patient issues that are of special importance to MICS.

\section{Cardiovascular}

In addition to the primary cardiac pathology, the presence of concomitant cardiac or vascular abnormalities has to be evaluated.

A thorough evaluation of patients' peripheral vasculature is necessary if peripheral bypass cannulation is planned $(2,3)$. The presence of plaques, aneurysms, dissections, tortuosity, or foreign vascular material (grafts or stents) might preclude peripheral cannulation. If endoballoon clamping is planned, higher degree of aortic valve insufficiency and aortic vascular disease, such as an aneurysmal ascending aorta or effacement of the sinotubular junction, must be excluded $(3,4)$. Many centers include CT angiography in the workup to MICS.

Impaired ventricular performance, patent foramen ovale (PFO), presence of a left superior vena cava (SVC) and other venous abnormalities can preclude or significantly alter the MICS technique.

\section{Respiratory}

Most MICS procedures performed via a thoracotomy involve SLV and require the patient to be positioned supine or with a slightly elevated hemithorax, both of which increase the risk of hypoxemia. A history of chronic lung disease, or reduced exercise capacity merits a formal evaluation of respiratory function and suitability for sustained SLV.

Forced 1 second expiration (FEV1) is a useful initial screening tool. Studies from thoracic surgical patients demonstrate that the risk of postoperative pulmonary complications increases with a FEV $1<40 \%$ of predicted (5).

Arterial blood gas sampling and carbon monoxide diffusion capacity (DLCO) are suggested to assess gas exchange. Traditionally, a $\mathrm{PaO}_{2}<60 \mathrm{mmHg}$ or a $\mathrm{PaCO}_{2}$ $>50 \mathrm{mmHg}$ preclude the use of one-lung ventilation (OLV) $(4,5)$, DLCO values $<40 \%$ are associated with increased incidence of postoperative pulmonary complications (5).

It is important to evaluate any degree of pulmonary hypertension and subsequent right ventricular (RV) dysfunction. Almost all patients will have a preoperative echocardiogram which should include an estimate of the right ventricular systolic pressure (RVSP), size and function. Pulmonary hypertension is not an absolute contraindication to SLV, but this technique can transiently increase pulmonary arterial pressure and RV afterload, which may precipitate failure and hemodynamic collapse. 
Previous radiotherapy, pleural adhesions, thoracic surgery, chest wall deformities, or rib trauma should also be identified, as they can impede surgical access.

\section{Gastrointestinal (GI)}

The ability to perform intraoperative TEE is integral to many MICS procedures, and a contraindication to TEE is a de facto contraindication to the surgical procedure itself. The presence of esophageal webs or strictures, tumor, laceration, diverticulum, or active upper GI bleeding are recognized absolute contraindications to TEE (6).

A history of radiation to the head neck or mediastinum, previous surgery of the upper GI tract, esophageal varices, active peptic ulcer disease, and hiatal hernia are among the most common relative contraindications to TEE (6). If any of these are present risks and benefits of TEE need to be assessed carefully.

\section{Musculoskeletal}

Patient positioning is an important part of MICS in order to optimize surgical exposure. This often involves positioning the patient with the operative hemithorax elevated. The ipsilateral arm is tucked which can place tension on the brachial plexus (7).

Morbidly obese patients pose a challenge for MICS, not least because of positioning for optimal surgical exposure. Patients should be screened for preexisting orthopedic or other musculoskeletal conditions, as they can have implications for both surgical and anesthetic technique.

\section{Surgical issues}

Although not an absolute contraindication, morbid obesity can make surgical access difficult and render the patient unsuitable for a MICS technique, especially in combination with very large breasts $(8,9)$. Additionally, very obese patients may require additional drainage cannula placement to provide higher CPB flow.

Chest wall deformities and kyphoscoliosis can complicate surgical access. Appropriate surgical exposure may be impossible in small patients with a small thoracic cavity. The size of their femoral vessels may preclude direct cannulation (8).

\section{Anesthetic issues}

Patients with chest wall deformities and kyphoscoliosis may have limited pulmonary reserve and may prove difficult to manage with SLV.
Placement of a double-lumen endotracheal tube (ETT) can be tricky in morbidly obese patients and they can have difficulty tolerating SLV. Although these patients present the biggest challenge for MICS, they may in fact benefit most from this approach as they are at increased risk for post-sternotomy complications (10).

\section{Intraoperative management}

\section{Setup}

Induction and conduct of anesthesia follow the same principles that apply to traditional cardiac surgery. The main differences are that in addition to potentially using a double-lumen ETT MICS is likely to require placement of external defibrillator pads as internal cardioversion is unavailable in the majority of procedures.

\section{Monitoring}

As is the case in conventional cardiac surgery, choice of monitoring during MICS is mostly institution and practitioner specific. It is highly advisable, however, to have bilateral radial arterial line monitoring if an endoballoon is being used, in order to detect balloon migration and occlusion of the brachiocephalic artery (2). An extra pressure transducer is necessary to monitor the pressure in the balloon tip.

If SLV is required, the presence of a unilateral pneumothorax may interfere with the electrocardiogram (ECG). Leads should be adjusted so that ECG monitoring is not disrupted (3).

Cerebral oximetry monitoring is useful if peripheral $\mathrm{CPB}$ is used. With this perfusion mode arterial blood is returned into the abdominal aorta and blood supply to the upper body occurs in a retrograde fashion. If during the period of no ventilation the heart is able to eject, oxygenated and deoxygenated blood will mix in the proximal aorta. This admixture, known as Harlequin syndrome, can result in differential hypoxemia of upper body tissues. Life-threatening cerebral hypoxemia can ensue if this mixing of blood occurs distal to the aortic arch. An abrupt fall in cerebral oximetry can help recognize this condition immediately (11).

\section{Vascular access}

As in conventional cardiac surgery, central venous access is necessary. The choice and location of catheter(s) is patient and procedure dependent. Bypass configuration, myocardial 
preservation strategy, and the need for venting or overdrive pacing all impact on line placement and need discussion within the clinical team. Details regarding vascular access for specific procedures will be discussed individually.

\section{Positioning}

After anesthetic induction and obtaining appropriate vascular access, the patient is carefully positioned. This is an integral part of many MICS operations and requires special attention from operating theatre staff to ensure that pressure points are sufficiently padded and that the neck, arms and legs are not hyperflexed or hyperextended. This must be done with meticulous care as it is difficult to access the patient after they have been prepped and draped.

\section{Cannulation}

Surgery begins with exposing the operating field, establishing arterial and venous access for CPB, venting and delivery of cardioplegia if necessary.

If CPB is required, venous cannulation is typically established first. This is most commonly achieved under TEE guidance with a single two-stage venous cannula, inserted in a femoral vein and advanced until the distal end is in the proximal SVC (12).

In surgery involving a right atriotomy a modification of the traditional bicaval technique is used. The inferior vena cava (IVC) cannula is placed femorally and advanced until it is just distal to the cavoatrial junction; the SVC cannula is placed in the right internal jugular vein via Seldinger technique and advanced proximal. This cannula is mostly inserted during the anesthetic induction under ultrasoundguidance alongside the central venous catheter. A bolus dose of 5,000 units of intravenous heparin is recommended prior to the insertion of the IVC cannula in order to prevent thrombus formation. The position of both cannulas is verified with TEE. Should venous drainage be inadequate with a femoral approach, an additional drainage cannula can be placed in the right or left internal jugular.

Arterial cannulation is usually established via one of the femoral arteries, using either an end-to-side graft, direct cut-down or Seldinger technique.

\section{Bypass and myocardial protection}

Excellent communication between surgeon, anesthesiologist and perfusionist throughout bypass is crucial.
After cannulation and when an appropriate surgical field has been established the patient is commenced on CBP and once flow is adequate the aorta is cross-clamped. This can be achieved through a number of different approaches:

* Under direct vision with a special device, typically a Chitwood Clamp, which requires a separate chest wall incision to insert. This device is capable of clamping ascending aortas of up to $45 \mathrm{~mm}$ in diameter (12). When using this method cardioplegia must be delivered into the proximal ascending aorta through a separate cannula.

* Using an endoballoon, which is typically inserted via a femoral or a subclavian artery. As the surgeon is guiding the balloon in place under TEE guidance the perfusionist regulates blood flow to guide the balloon proximally into its position in the ascending aorta. Proper balloon position needs to be carefully verified by TEE before inflation. If the balloon is placed too distally the innominate artery may be occluded resulting in cerebral and limb ischemia; if the balloon is placed too proximally cardioplegia may not reach the coronary ostia (12). When the endoballoon is fully inflated the aortic wall will deform slightly to form a tight seal, preventing systemic flow of cardioplegia solution. Abnormal aortic anatomy may prevent a sufficient seal resulting in inadequate myocardial protection. Cardioplegia is delivered through the tip of the balloon.

TEE can help ensure that myocardial protection is adequate by visualization of cardioplegia flow in the left and right coronary arteries. Left ventricular (LV) distension during administration of cardioplegia can also be identified.

After the surgical procedure is completed, the patient is liberated from CPB in a similar manner as in conventional cardiac surgery. Although SLV may be required for hemostasis before chest closure, temporarily expanding both lungs is useful for weaning from CPB by optimizing not only ventilation and oxygenation but also RV performance.

\section{Transesophageal echocardiography}

In addition to the standard indications for intraoperative TEE, the majority of MICS procedures rely on TEE. Table 1 summarizes the points any comprehensive TEE examination must address.

Erroneous CPB cannula positioning can have devastating consequences such as inadvertent cannulation of the hepatic veins or perforation of the right atrium, right ventricle or 
Table 1 Mandatory points to be addressed by intraoperative TEE

Rule out significant aortic valve regurgitation

Asses size and morphology of the aortic root and ascending aorta

Advise of any atheromatous disease in the aorta

Inform of the presence of a PFO or ASD

Verify correct CPB cannula placement

Guide endoballoon positioning

Guide positioning of additional lines like pulmonary artery vents or coronary sinus catheters if used

TEE, transesophageal echocardiography; PFO, patent foramen ovale; ASD, atrial septal defect; CPB, cardiopulmonary bypass.

interatrial septum.

Endoballoon visualization in the proximal ascending aorta can be obscured by the left mainstem bronchus. If a double-lumen ETT is used, inflating the bronchial cuff with saline can provide an acoustic window to better see the balloon position. Cardioplegia flow into the left and right coronary artery can be seen using color flow doppler.

The small incisions used in MICS do not afford the anesthesiologist and the surgeon a complete view of the right ventricle, as is the case in a conventional sternotomy. TEE is the pivotal tool to assess RV size and performance.

TEE may be important to assess distension of the left ventricle. During cardioplegia administration, the presence of aortic regurgitation can result in $\mathrm{LV}$ distension and inadequate myocardial protection. New onset of aortic valve regurgitation can occur as a result of migration of the endoballoon or distortion of the aortic annulus after balloon inflation. Inadequate venting of the left-sided heart chambers can also result in $\mathrm{LV}$ distension.

\section{Intraoperative events}

If cardioversion or defibrillation is necessary, it must be delivered externally. If the patient is single lung ventilated at the time the electrical impedance between the defibrillator pads may be too high to deliver a sufficient electrical current and the collapsed lung might have to be reinflated temporarily.

Emergency conversion to full sternotomy occurs in $2-3 \%$ of cases $(13,14)$. The most frequent reason is excessive bleeding, which is associated with a mortality rate upwards of $20 \%$. Other reasons for conversion include poor exposure of the mitral valve, adhesions, and iatrogenic aortic dissection (12). Should emergency conversion be required, extra personnel will likely be needed in order to simultaneously manage massive transfusion and reposition the patient. Ongoing excellent communication between anesthetic, surgical and perfusion teams is vital during this period. Should deep hypothermic circulatory arrest (DHCA) become necessary in case of aortic injury extra measures such as head cooling or additional cannulation sites add to the complexity.

\section{Analgesia}

Intravenous opioids are often the mainstay of analgesia for cardiac surgical procedures. However, regional anesthesia can play a significant role in facilitating postoperative pain relief in MICS. Blocks can be performed before surgery in order to minimize intraoperative opioid requirements, or at the conclusion of surgery in order to maximize the duration of postoperative pain relief. The choice of regional or neuraxial anesthesia depends on institutional as well as individual practitioners' experience and on the surgical approach.

\section{Opioid sparing and opioid free anesthesia (OFA)}

Opioids over the decades have been one of the corner stones of a 'balanced cardiac anesthetic technique', providing analgesia as well as avoiding a sympathetic response during cardiac surgery. Although an effective analgesic, they come with a myriad of side effects including opioid induced respiratory depression, delirium, hyperalgesia, constipation or urinary retention, all of which can hamper patients' recovery and lead to unwarranted morbidity (15). Enhanced recovery after surgery (ERAS) protocols in non-cardiac surgery have led the way in gradually moving away from large dose opioids in the perioperative period and OFA is gaining popularity in the general surgical specialties (16). The concept of OFA rests on maintaining hypnosis and muscle relaxation using traditional means, while providing analgesia and sympatholysis using a combination of adjuncts such as ketamine, magnesium, alpha1-receptor agonists like dexmedetomidine or inhalational agents and nerve blocks while aiming to avoid central neuraxial blockade.

In recent years cardiac anesthesiologists have also started moving away from giving large doses of opioids towards more conservative doses (17). The innervation of the chest wall lends to peripheral nerve blockade, thereby providing cardio-stable conditions as well as good analgesia. This along with a combination adjunct drugs can provide 
Table 2 Neuraxial and loco-regional techniques for postoperative analgesia

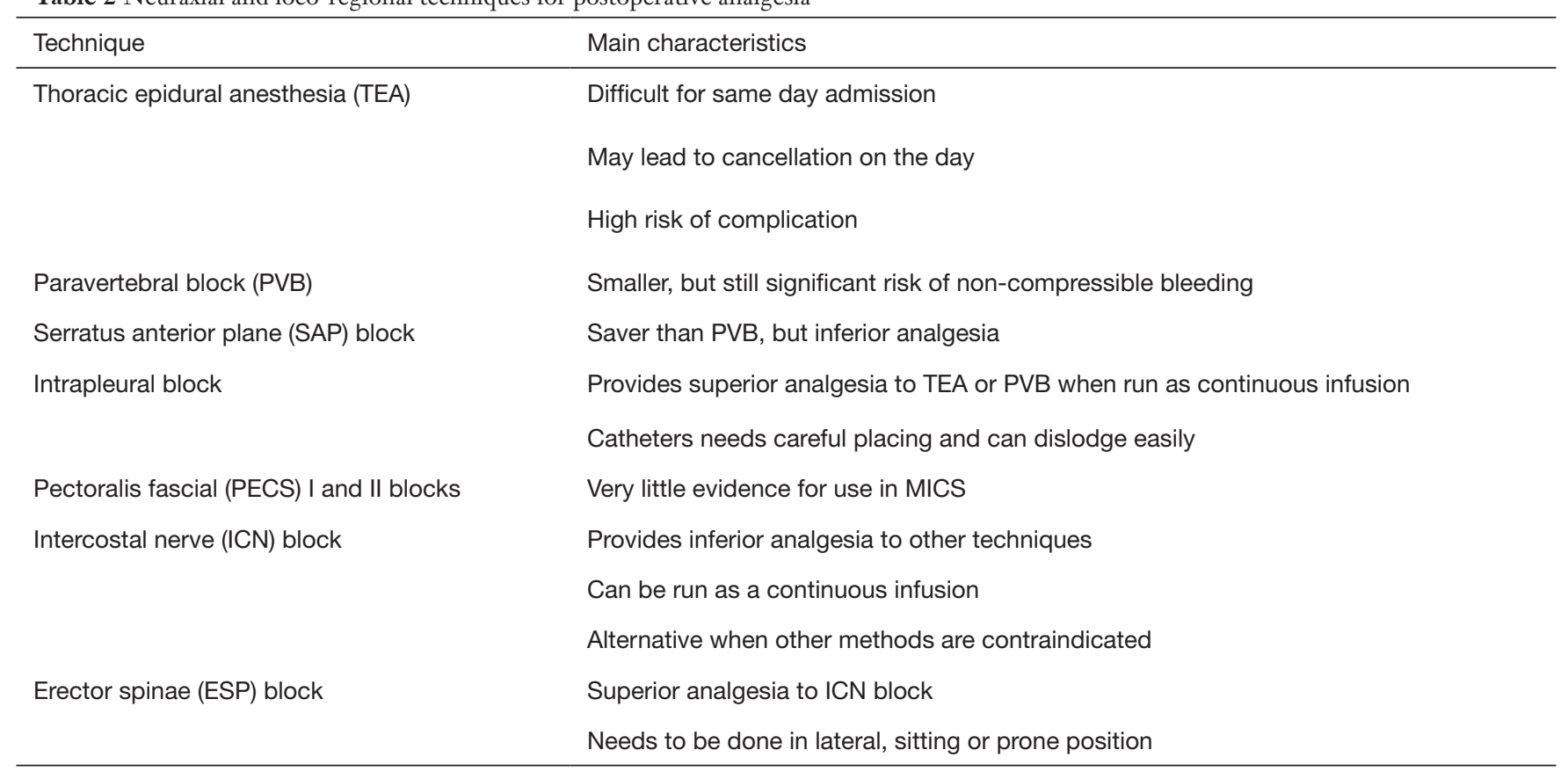

MICS, minimal invasive cardiac surgery.

effective and safe perioperative conditions for cardiac. With an understanding of the pharmacokinetics of these drugs, it is possible to appropriately time their discontinuation to facilitate early or even extubation on table when clinically appropriate.

Combining an OFA technique with non-opioid based postoperative analgesia using drugs such as pregabalin or paracetamol can allow creating an effective 'opioid free' or relatively 'opioid sparing' recovery environment (18). The success of this is largely dependent on using locoregional techniques, often as a continuous local anesthetic infusion through an intraoperatively placed catheter, during the initial recovery period (19). Currently clinical trials are underway to assess the benefit and safety of OFA in cardiac surgery and will in due course answer some of the questions about safety and effectiveness of this novel technique (20).

Minimally invasive cardiac surgery access is in most cases amenable to local anesthesia techniques, which in turn helps with realizing the potential benefits of early extubation and early mobilization. Table 2 summarizes the regional techniques suitable for MICS and their main characteristics.

\section{Thoracic epidural analgesia (TEA)}

TEA is well-described in the cardiac anesthetic literature. Its use in MICS has been less studied, however one small study in minimally invasive coronary bypass surgery demonstrated less postoperative pain and decreased hospital stay (21). The catheter is usually inserted between T4 and $\mathrm{T} 7$, although a cervical approach has been described in a number of publications. Both approaches provide analgesia to most MICS incision sites.

There is of course significant concern about the development of an epidural hematoma. The American Society of Regional Anesthesia guidelines (22) suggest that neuraxial anesthesia is contraindicated in a patient with a known coagulopathy from any cause. They suggest that systemic heparinization should occur no sooner than 60 minutes after instrumentation, and that surgery should be delayed for 24 hours in the event of a traumatic tap making it difficult to use this technique in same-day admission surgery. The overall risk of epidural hematoma is approximately 1:3,500 (23). These drawbacks and safety concerns have limited the widespread use of TEA in cardiac anesthesia and are likely to continue to do so, also in the emerging field of MICS.

\section{Thoracic paravertebral block (TPB)}

A paravertebral block (PVB) should be placed at the T2T4 level as the paravertebral space is generally largest there and thus minimizes the risk of causing a pneumothorax. 
There is less risk of epidural hematoma formation, particularly when using ultrasound, comparted to TEA, and it can be performed relatively quickly $(24,25)$. It has been demonstrated to reduce intraoperative and postoperative narcotic usage, lower pain scores and is as effective as TEA (26).

PVBs have also been used in transapical (TA) transcatheter aortic valve implantation (TAVI) and result in less opioid usage as well as decrease in AF rates while maintaining a smooth hemodynamic profile $(27,28)$.

There is some risk of noncompressible bleeding with the TPB block although the risk of neurological injury is lower than TEA.

\section{Serratus anterior plane (SAP) block}

The SAP block is performed in a much more lateral plane than a PVB, and therefore does not cause a sympathectomy and may have a better safety profile in patients who are anticoagulated (24).

One French study compared the SAP block to a PVB in patients receiving minimally invasive coronary bypass surgery and found that postoperative opioid consumption was higher in patients receiving an SAP block, suggesting that although it is theoretically safer, there may be a tradeoff in terms of analgesic efficacy (29).

\section{Intrapleural block}

Intrapleural analgesia is usually provided by inserting a catheter between the visceral and parietal pleura, and is often done intraoperatively by the surgical team under direct vision (18). When compared to TEA in minimally invasive coronary bypass surgery, intrapleural analgesia was found to provide superior pain relief. Although a simple and safe alternative to TEA, intrapleural catheters require careful positioning and manipulation of chest tubes in order to be fully effective (30).

\section{Pectoralis fascial (PECS) I and II blocks}

The PECS I block targets the medial and lateral pectoral nerves and may block the intercostobrachial nerve and the intercostal nerve (ICN) branches anteriorly (31). The PECS II block targets the long thoracic nerve and thoracodorsal nerve (31). The literature evaluating PECS blocks for MICS is sparse, there are several reports of it being used with varying success in TA TAVI procedures (32).

Erector spinae (ESP) block
In an ESP block, local anesthetic is injected ventral to the erector spine muscle and targets the ventral and dorsal rami of spinal nerves. This provides analgesia to the sternum, as well as the medial and lateral regions of the chest wall (31). The ESP block has been shown to provide superior pain relief to systemic analgesia in patients undergoing conventional cardiac surgery and reduce intraoperative opioid consumption (33). It has recently been shown to provide superior analgesia for MICS when compared to intercostal blocks placed under direct vision (34). The ESP block is performed in lateral, sitting or prone position which may limit its practicality (35).

\section{ICN block}

ICN blockade can be performed by the anesthesiologist, or under direct vision by the surgical team (36). The plane is well-suited for catheter insertion to provide a continuous infusion of local anesthesia. Patients undergoing TA TAVI had a higher pain score with ICN blocks compared to TEA, although it still provided effective pain control. ICN catheters provide a reasonably safe alternative for patients who have a contraindication to neuraxial blockade or where is technique is best avoided (37).

\section{Postoperative care}

Patients undergoing MICS require admission to a cardiothoracic intensive care unit. A double-lumen ETT should be changed for a single lumen tube before transfer. Cannulation sites for peripheral CPB cannulation have to be monitored for hematoma, thrombus, and pseudoaneurysm formation, and the integrity of limb perfusion distal to the cannulation sites has to be assessed regularly.

If there are no major complications, MICS patients are typically placed on a "fast track" protocol, weaned from sedation and extubated as soon as it is appropriate. Some institutions prefer "ultra-fast tracking" with extubation in the operating theatre.

\section{Procedure specific considerations}

Despite the non-traditional surgical approach, the management of MICS patients still follows the same principles as conventional cardiac surgery. In addition to the general considerations for MICS there are some particularities to each individual procedure. 


\section{Minimally invasive coronary artery bypass grafting (MIDCABG)}

MIDCABG is typically performed via a left thoracotomy approach, the exact location of the thoracotomy will depend on the coronary artery distribution. A midline approach via an inferior mini-sternotomy may be used. Some centers prefer total endoscopic techniques. Depending on the anatomy and surgical approach, the procedure can be performed on $\mathrm{CPB}$, or with the heart beating (OPMIDCABG).

As in conventional surgery, an OP-MIDCABG requires inotropes and vasopressors to be readily available in order to compensate for the rapid and sometimes profound hemodynamic changes that accompany cardiac manipulation. Depending on the incision and procedure, a combination of any of intercostal, PECS I and II, pecto-intercostal fascial (PIF), transverse thoracic muscle plane (TTMP), and SAP nerve block techniques may be employed.

\section{Minimally invasive mitral valve surgery (MIMVS)}

MIMVS is most commonly performed via a right thoracotomy (12). The main contraindications are a previous right thoracotomy, severe aortic calcification, mitral annular calcification, aortic root or ascending aorta dilation. As several parts of the procedure are highly dependent on echocardiography any contraindication to TEE should be considered a contraindication to MIMVS.

Circumflex artery occlusion is a rare but serious complication of mitral valve surgery, and there may be an increased risk in MIMVS. Patency of the circumflex artery can be demonstrated by TEE (38).

Analgesia is typically targeted at controlling thoracotomy pain, which can be achieved with any of thoracic paravertebral (TPV), ESP, SAP, or ICN blockade.

\section{Minimally invasive aortic surgery}

The most common approaches for minimally invasive aortic valve surgery (mAVR) are an upper partial sternotomy, about one third the size of a conventional sternotomy, or a right anterior mini-thoracotomy. Patients undergoing mAVR via a mini-thoracotomy should be able to tolerate OLV. Typically, arterial cannulation is central, while venous cannulation is often femoral.

Different analgesia options exist depending on the surgical approach. If mAVR surgery is performed via an upper sternotomy, analgesia can be achieved via a PECS I and II block, a transversus thoracic plane (TTP) block, or an ESP block; if the approach is via a mini-thoracotomy, analgesia can be achieved with SAP, paravertebral or intercostal block.

\section{Minimally invasive surgery for correction of $A F$}

Surgical AF correction may involve a variation on the traditional Maze procedure, pulmonary vein isolation, and left atrial appendage (LAA) excision and is generally done off $\mathrm{CPB}$ via uni- or bilateral video-assisted thoracoscopic surgery (VATS). Unlike traditional VATS, however, the patient is in a supine or semi-supine and steep reverse Trendelenburg position.

Intraoperative TEE, although not mandatory, can be used to assess the left atrium for thrombus and the pulmonary veins for stenosis post procedure.

Analgesia is best achieved intraoperatively by the surgical team via ICN blocks under direct visualization. Paravertebral or ESP blocks can also be effective. Thoracic epidural catheterization is possible but not advisable given the need for perioperative anticoagulation, and the potential need for systemic heparinization and emergency bypass.

\section{Angiography suite/hybrid theatre procedures}

Many cardiac interventions which traditionally required open surgery can now in selected patients be done with transcatheter techniques. These provide an alternative to standard surgery on CPB, which may be deemed too risky. It is important to have a discussion with the patient as well as the cardiology and surgical teams about how to proceed in the event of an intraoperative complication which could potentially require $\mathrm{CPB}$ and a more invasive surgical approach. Because of their underlying frailty, many patients are not considered for escalation of support or invasiveness.

One of the most commonly performed transcatheter cardiac interventions is TAVI. Although the vast majority of these is performed without anesthetic or surgical input using the transfemoral approach, there is still a place for mini-thoracotomy, TA and increasingly transcarotid (TC) TAVI. They are chosen when unfavorable anatomy of the aorta, femoral or iliac arteries preclude a transfemoral approach. Patients for TC TAVI should have an intact Circle of Willis and favorable carotid anatomy (39). Their ability to cooperate needs to be assessed very carefully if a 
TC TAVI under local anesthetic is considered. Converting to general anesthetic mid-procedure is very difficult and can give rise to a number of new problems due to the proximity of the surgical field and the airway. TC TAVI under local anesthetic may increase in popularity after a retrospective cohort study demonstrated a higher rate of perioperative stroke with general anesthesia (40).

\section{Intraoperative conduct}

TAVIs are usually conducted in hybrid operating theatres, with fluoroscopic capabilities. Transthoracic echocardiography (TTE) is the ultrasound modality of choice. TEE is an alternative but is only used in intubated patients if transthoracic imaging is too poor to allow adequate visualization.

Routine monitors include percutaneous defibrillation pads and cerebral $\mathrm{O}_{2}$ saturation. Discussion as to laterality of the 'anesthetic' arterial line is important because the interventional team requires arterial access for the intraoperative angiography. The interventional team typically places a femoral central venous line with a sheath introducer to place a temporary pacing wire into the right ventricle. The sidearm of the sheath affords central venous access for the anesthesiologist. If a separate central venous line is required, the left internal jugular should be avoided in TC TAVI patients as it will be in the operative field. TA TAVIs can either be done using a double-lumen ETT with right SLV or using a supraglottic airway and spontaneous ventilation. Our institutional experience shows that both afford the surgeon equal view of and access to the left ventricular apex.

After anesthetic induction and once arterial and venous access necessary for the procedure have been established, the bioprosthetic aortic valve is implanted by crossing the diseased valve with a guidewire followed by balloon dilation and valve deployment. Cardiac standstill during dilatation and valve implantation is achieved by temporary rapid ventricular pacing. Angiography and TTE or TEE are used to assess valve placement and function.

There can be significant hemodynamic disturbances during and after the valve implantation and poor $\mathrm{LV}$ function may persist. Vasopressors and inotropes may be required to support the patient's hemodynamics during this period. Other complications include complete heart block, pericardial tamponade, and ischemia from coronary ostial obstruction (41). Valve-in-valve patients are at particularly high risk for coronary ostial obstruction by the preexisting tissue aortic valve leaflets (42). Aortic regurgitation can occur if there is a perivalvular leak. Rarely the valve may embolize.

Echocardiography after deployment assesses correct valve placement, the presence of a paravalvular leak, mitral leaflet motion, and can detect the presence of new wall motion abnormalities. Any prolonged post-procedural hemodynamic perturbation should prompt TTE screening to exclude tamponade. Heart block may occur as a result of valve deployment, and temporary transvenous pacing may be required. Some patients will need to have a permanent pacemaker fitted (43).

\section{Analgesia}

As it is done through a mini-thoracotomy the postoperative pain associated with TA TAVI is often more severe than after other TAVI approaches (44). There are a number of regional techniques to provide analgesia for a TA TAVI approach. The use of a TPB has been shown to provide effective analgesia and reduce opioid administration, rates of postoperative $\mathrm{AF}$, and lead to earlier extubation time. Although less studied, the SAP blockade is typically employed at our institution and has been described as an alternative to general anesthesia for TA TAVI (45).

A superficial cervical plexus block can provide good analgesia postoperatively after TC TAVI. Supplemental pain control can be obtained via patient controlled IV anesthesia.

\section{Postoperative considerations}

Patients are usually extubated in the operating theatre. If there is no need for ongoing respiratory or hemodynamic support, patients can be admitted to a post-anesthesia care unit (PACU) for monitoring. Particular attention needs to be paid to the vascular access sites and to early detection of signs of cardiac ischemia or tamponade. A higher-grade heart block may occur. Perioperative stroke is a well-known complication of TAVI procedures, and the rate has been reported at $2-5 \%$, depending on the mode of access (46-48).

Bleeding complications may occur at the vascular access site, including hemorrhage, dissection and pseudoaneurysm. Rates vary widely depending on the site and the surgical technique, as well as patient risk factors and range between 4-25\% (41,49,50). TC TAVI has the lowest postoperative bleeding rate, injury at the carotid vascular access site appears to be vanishingly rare (51). Careful neurologic 
monitoring is the mainstay of postoperative management for these patients, although perioperative strokes appear to be rare and transient in nature $(40,51)$.

Patients are typically transferred to a regular ward if there are no complications.

\section{Discussion}

The conduct of MICS is inherently more complicated for both surgeons and anesthesiologists. Prolonged surgical and CPB times are consistently seen with MICS (5254). However, this may be offset by a number of potential benefits. A reduction in intraoperative bleeding and transfusion rates has been demonstrated $(14,52,55)$, as well as decreased ICU and hospital length of stay $(53,56)$. Observational studies have also reported an improvement in postoperative pain scores (56), particularly with the increased use of regional anesthesia techniques.

Although there is a paucity of large randomized data, results demonstrate that important clinical outcomes are at least equivalent between to conventional approaches. Rates of mortality, stroke, acute kidney injury, aortic dissection, $\mathrm{AF}$ and 30-day readmission are equivalent (53,56-62). For valvular surgery, repair rates are equal among those undergoing MICS vs. conventional cardiac surgery (52). The best outcomes of MICS are realized with a thorough preoperative evaluation, an anesthetic team skilled in TEE and multimodal analgesia, and an experienced surgical team.

The literature is rife with trials comparing outcomes between a surgical approach to aortic valve replacement (SAVR) with the TAVI approach. Four landmark trials comparing these approaches (63-66) were analyzed in a 2016 meta-analysis (67) and revealed that the transfemoral TAVI approach resulted in significantly lower mortality than SAVR across high, intermediate and low surgical risk groups, however this reduction was not seen in with a transthoracic TAVI approach. TC TAVIs were not included in the analysis. The meta-analysis also revealed a lower incidence of acute kidney injury, new-onset AF and major bleeding among the TAVI population, while showing higher rates of major vascular complications, need for permanent pacemaker, and paravalvular regurgitation.

\section{Conclusions}

Minimally invasive cardiac surgery encompasses a wide range of surgical procedures both in operating theatres and hybrid catheterization laboratories. The potential benefits are many and include reduced pain and hospital stay as well as increased patient satisfaction. Furthermore, the advances in minimally invasive and transcatheter approaches are allowing patients to have life-saving operations, where they would have otherwise been deemed too high-risk for a traditional approach.

In order for these benefits to be properly realized, the anesthesiologist must be equipped to face the unique challenges they present. This includes a thorough and comprehensive preoperative assessment, a high degree of intraoperative vigilance, proficiency with TEE and multi-modal analgesia and most importantly excellent communication with the perfusionist and surgical team.

\section{Acknowledgments}

Funding: None.

\section{Footnote}

Provenance and Peer Review: This article was commissioned by the Guest Editors (Jason Ali and Yasir Abu-Omar) for the series "Minimally Invasive Cardiac Surgery" published in Fournal of Thoracic Disease. The article was sent for external peer review organized by the Guest Editors and the editorial office.

Peer Review File: Available at http://dx.doi.org/10.21037/jtd20-1804

Conflicts of Interest: All authors have completed the ICMJE uniform disclosure form (available at http://dx.doi. org/10.21037/jtd-20-1804). The series "Minimally Invasive Cardiac Surgery" was commissioned by the editorial office without any funding or sponsorship. The authors have no other conflicts of interest to declare.

Ethical Statement: The authors are accountable for all aspects of the work in ensuring that questions related to the accuracy or integrity of any part of the work are appropriately investigated and resolved.

Open Access Statement: This is an Open Access article distributed in accordance with the Creative Commons Attribution-NonCommercial-NoDerivs 4.0 International License (CC BY-NC-ND 4.0), which permits the noncommercial replication and distribution of the article with the strict proviso that no changes or edits are made and the 
original work is properly cited (including links to both the formal publication through the relevant DOI and the license). See: https://creativecommons.org/licenses/by-nc-nd/4.0/.

\section{References}

1. Rosengart TK, Feldman T, Borger MA, et al. Percutaneous and minimally invasive valve procedures: a scientific statement from the American Heart Association Council on Cardiovascular Surgery and Anesthesia, Council on Clinical Cardiology, Functional Genomics and Translational Biology Interdisciplinary Working Group, and Quality of Care and Outcomes Research Interdisciplinary Working Group. Circulation 2008;117:1750-67.

2. Knowles AC, Coddens J. Minimally invasive cardiac surgery. In: Mackay JH, Arrowsmith JE. editors. Core topics in cardiac anesthesia. 2nd ed. Cambridge: Cambridge University Press, 2012.

3. Malik V, Jha AK, Kapoor PM. Anesthetic challenges in minimally invasive cardiac surgery: Are we moving in a right direction? Ann Card Anaesth 2016;19:489-97.

4. Balasubramanyam U, Kapoor PM. Anesthetic challenges in minimally invasive cardiac surgery. J Card Crit Care TSS 2020;3:28-35.

5. Slinger P, Darling G. Preanesthetic assessment for thoracic surgery. In: Slinger P. editor. Principles and practice of anesthesia for thoracic surgery. Cham: Springer, 2019.

6. Hahn RT, Abraham T, Adams MS, et al. Guidelines for performing a comprehensive transesophageal echocardiographic examination: recommendations from the American Society of Echocardiography and the Society of Cardiovascular Anesthesiologists. J Am Soc Echocardiogr 2013;26:921-64.

7. Vernick W, Alturi P. Robotic and minimally invasive cardiac surgery. Anesthesiol Clin 2013;31:299-320.

8. Müller L, Höfer D, Holfeld J, et al. Indications and contra-indications for minimally invasive mitral valve surgery. J Vis Surg 2018;4:255.

9. Klein P, Klop IDG, Kloppenburg GLT, et al. Planning for minimally invasive aortic valve replacement: key steps for patient assessment. Eur J Cardiothorac Surg 2018;53:ii3-8.

10. Reser D, Sündermann S, Grünenfelder J, et al. Obesity should not deter a surgeon from selecting a minimally invasive approach for mitral valve surgery. Innovations (Phila) 2013;8:225-9.

11. Kanda H, Kunisawa T, Kitahara H, et al. Cerebral hypoxia caused by flow confliction during minimally invasive cardiac surgery with retrograde perfusion: a word of caution. J Cardiothorac Vasc Anesth. 2018;32:1838-40.

12. Parnell A, Prince M. Anaesthesia for minimally invasive cardiac surgery. BJA Education 2018;18:323-30.

13. Salenger R, Gammie JS, Collins JA. Minimally invasive aortic valve replacement. J Card Surg 2016;31:38.

14. Falk V, Cheng DC, Martin J, et al. Minimally invasive versus open mitral valve surgery: a consensus statement of the international society of minimally invasive coronary surgery (ISMICS) 2010. Innovations (Phila) 2011;6:66-76.

15. Lavand'homme P, Steyaert A. Opioid-free anesthesia opioid side effects: tolerance and hyperalgesia. Best Pract Res Clin Anaesthesiol 2017;31:487-98.

16. Forget P. Opioid-free anaesthesia. Why and how? A contextual analysis. Anaesth Crit Care Pain Med 2019;38:169-72.

17. Rong LQ, Kamel MK, Rahouma M, et al. High-dose versus low-dose opioid anesthesia in adult cardiac surgery: a meta-analysis. J Clin Anesth 2019;57:57-62.

18. Landry E, Burns S, Pelletier MP, et al. A successful opioidfree anesthetic in a patient undergoing cardiac surgery. $\mathrm{J}$ Cardiothorac Vasc Anesth 2019;33:2517-20.

19. Chanowski EJ, Horn JL, Boyd JH, et al. Opioid-free ultrafast-track on-pump coronary artery bypass grafting using erector spinae plane catheters. J Cardiothorac Vasc Anesth 2019;33:1988-90.

20. Beloeil H, Laviolle B, Menard C, et al. POFA trial study protocol: a multicentre, double-blind, randomised, controlled clinical trial comparing opioid-free versus opioid anaesthesia on postoperative opioid-related adverse events after major or intermediate non-cardiac surgery. BMJ Open 2018;8:e020873.

21. Kurtoğlu M, Ateş S, Bakkaloğlu B, et al. Epidural anesthesia versus general anesthesia in patients undergoing minimally invasive direct coronary artery bypass surgery. Anadolu Kardiyol Derg 2009;9:54-8.

22. Horlocker TT, Vandermeuelen E, Kopp SL, et al. Regional anesthesia in the patient receiving antithrombotic or thrombolytic therapy: American Society of Regional Anesthesia and Pain Medicine Evidence-Based Guidelines. Reg Anesth Pain Med 2018;43:263-309.

23. Landoni G, Isella F, Greco M, et al. Benefits and risks of epidural analgesia in cardiac surgery. Br $\mathrm{J}$ Anaesth 2015;115:25-32.

24. Yu S, Valencia MB, Roques V, et al. Regional analgesia for minimally invasive cardiac surgery. J Card Surg 2019;34:1289-96.

25. Neuburger PJ, Ngai JY, Chacon MM, et al. A prospective 
randomized study of paravertebral blockade in patients undergoing robotic mitral valve repair. J Cardiothorac Vasc Anesth 2015;29:930-6.

26. Dhole S, Mehta Y, Saxena H, et al. Comparison of continuous thoracic epidural and paravertebral blocks for post-operative analgesia after minimally invasive direct coronary artery bypass surgery. J Cardiothorac Vasc Anesth 2001;15:288-92.

27. Okitsu K, Iritakenishi T, Iwasaki M, et al. Paravertebral block decreases opioid administration without causing hypotension during transapical transcatheter aortic valve implantation. Heart Vessels 2016;31:1484-90.

28. Poltak JM, Cobey FC, Augoustides JG, et al. Paravertebral analgesia in transapical transcatheter aortic valve replacement. Heart Lung Vessel 2015;7:217-23.

29. Moll V, Maffeo C, Mitchell M, et al. Association of serratus anterior plane block for minimally invasive direct coronary artery bypass surgery with higher opioid consumption: a retrospective observational study. J Cardiothorac Vasc Anesth 2018;32:2570-7.

30. Mehta Y, Swaminathan M, Mishra Y, et al. A comparative evaluation of intrapleural and thoracic epidural analgesia for post-operative pain relief after minimally invasive direct coronary artery bypass surgery. J Cardiothorac Vasc Anesth 1998;12:162-5.

31. Kelava M, Alfirevic A, Bustamante S, et al. Regional anesthesia in cardiac surgery: an overview of fascial plane chest wall blocks. Anesth Analg 2020;131:127-35.

32. Shakuo T, Kakumoto S, Kuribayashi J, et al. Continuous PECS II block for postoperative analgesia in patients undergoing transapical transcatheter aortic valve implantation. JA Clin Rep 2017;3:65.

33. Krishna SN, Chauhan S, Bhoi D, et al. Bilateral erector spinae plane block for acute post-surgical pain in adult cardiac surgical patients: a randomized controlled trial. J Cardiothorac Vasc Anesth 2019;33:368-75.

34. Fiorelli S, Leopizzi G, Menna C, et al. Ultrasound-guided erector spinae plane block versus intercostal nerve block for post-minithoracotomy acute pain management: a randomized controlled trial. J Cardiothorac Vasc Anesth 2020;34:2421-9.

35. Macaire P, Ho N, Nguyen T, et al. Ultrasound-guided continuous thoracic erector spinae plane block within an enhanced recovery program is associated with decreased opioid consumption and improved patient postoperative rehabilitation after open cardiac surgery-a patientmatched, controlled before-and-after study. J Cardiothorac Vasc Anesth 2019;33:1659-67.
36. Ueshima H, Otake H. Continuous transversus thoracic muscle plane block is effective for the median sternotomy. J Clin Anesth 2017;37:174.

37. Amat-Santos IJ, Dumont E, Villeneuve J, et al. Effect of thoracic epidural analgesia on clinical outcomes following transapical transcatheter aortic valve implantation. Heart 2012;98:1583-90.

38. Ender J, Selbach M, Borger MA, et al. Echocardiographic identification of iatrogenic injury of the circumflex artery during minimally invasive mitral valve repair. Ann Thorac Surg 2010;89:1866-72.

39. Pour-Ghaz I, Raja J, Bayoumi M, et al. Transcatheter aortic valve replacement with a focus on transcarotid: a review of the current literature. Ann Transl Med 2019;7:420.

40. Debry N, Delhaye C, Azmoun A, et al. Transcarotid transcatheter aortic valve replacement: general or local anesthesia. JACC Cardiovasc Interv 2016;9:2113-20.

41. Généreux P, Head SJ, Van Mieghem NM, et al. Clinical outcomes after transcatheter aortic valve replacement using valve academic research consortium definitions: a weighted meta-analysis of 3,519 patients from 16 studies. J Am Coll Cardiol 2012;59:2317-26.

42. Webb JG, Dvir D. Transcatheter aortic valve replacement for bioprosthetic aortic valve failure: the valve-in-valve procedure. Circulation 2013;127:2542-50.

43. Chacko M, Weinberg L. Aortic valve stenosis: perioperative anaesthetic implications of surgical replacement and minimally invasive interventions. Continuing Education in Anaesthesia Critical Care \& Pain 2012;12:295-301.

44. Mittnacht AJC, Shariat A, Weiner MM, et al. Regional techniques for cardiac and cardiac-related procedures. J Cardiothorac Vasc Anesth 2019;33:532-46.

45. Berthoud V, Ellouze O, Bièvre T, et al. Serratus anterior plane block for apical TAVR in an awake patient. J Cardiothorac Vasc Anesth 2018;32:2275-7.

46. Holmes DR Jr, Mack MJ, Kaul S, et al. 2012 ACCF/ AATS/SCAI/STS expert consensus document on transcatheter aortic valve replacement. J Am Coll Cardiol 2012;59:1200-54.

47. Daneault B, Kirtane AJ, Kodali SK, et al. Stroke associated with surgical and transcatheter treatment of aortic stenosis: a comprehensive review. J Am Coll Cardiol 2011;58:2143-50.

48. Moat NE, Ludman P, de Belder MA, et al. Long-term outcomes after transcatheter aortic valve implantation in high-risk patients with severe aortic stenosis: the U.K. 
TAVI (United Kingdom Transcatheter Aortic Valve Implantation) Registry. J Am Coll Cardiol 2011;58:2130-8.

49. Tomey MI, Mehran R. Bleeding avoidance in transcatheter aortic valve replacement: a call to ACTion? JACC Cardiovasc Interv 2014;7:152-3.

50. Rodés-Cabau J, Dauerman HL, Cohen MG, et al. Antithrombotic treatment in transcatheter aortic valve implantation: insights for cerebrovascular and bleeding events. J Am Coll Cardiol 2013;62:2349-59.

51. Mylotte D, Sudre A, Teiger E, et al. Transcarotid transcatheter aortic valve replacement: feasibility and safety. JACC Cardiovasc Interv 2016;9:472-80.

52. Moscarelli M, Fattouch K, Casula R, et al. What is the role of minimally invasive mitral valve surgery in highrisk patients? A meta-analysis of observational studies. Ann Thorac Surg 2016;101:981-9.

53. Cao C, Gupta S, Chandrakumar D, et al. A meta-analysis of minimally invasive versus conventional mitral valve repair for patients with degenerative mitral disease. Ann Cardiothorac Surg 2013;2:693-703.

54. Nair SK, Sudarshan CD, Thorpe BS, et al. Mini-Stern Trial: a randomized trial comparing mini-sternotomy to full median sternotomy for aortic valve replacement. J Thorac Cardiovasc Surg 2018;156: 2124-32.e31.

55. Kirmani BH, Jones SG, Malaisrie SC, et al. Limited versus full sternotomy for aortic valve replacement. Cochrane Database Syst Rev 2017;4:CD011793.

56. Modi P, Hassan A, Chitwood Jr WR. Minimally invasive mitral valve surgery: a systematic review and metaanalysis. Eur J Cardiothorac Surg 2008;34:943-52.

57. Speziale G, Nasso G, Esposito G, et al. Results of mitral valve repair for Barlow disease (bileaflet prolapse) via right minithoracotomy versus conventional median sternotomy: a randomized trial. J Thorac Cardiovasc Surg 2011;142:77-83.

Cite this article as: White A, Patvardhan C, Falter F. Anesthesia for minimally invasive cardiac surgery. J Thorac Dis 2021;13(3):1886-1898. doi: 10.21037/jtd-20-1804
58. Johnston WF, Ailawadi G. Surgical management of minimally invasive aortic valve operations. Semin Cardiothorac Vasc Anesth 2012;16:41-51.

59. McClure RS, Cohn LH, Wiegerinck E, et al. Early and late outcomes in minimally invasive mitral valve repair: an eleven-year experience in 707 patients. J Thorac Cardiovasc Surg 2009;137:70-5.

60. Galloway AC, Schwartz CF, Ribakove GH, et al. A decade of minimally invasive mitral repair: long-term outcomes. Ann Thorac Surg 2009;88:1180-4.

61. Mohr FW, Falk V, Diegeler A, et al. Computer-enhanced "robotic" cardiac surgery: experience in 148 patients. J Thorac Cardiovasc Surg 2001;121:842-53.

62. Suri RM, Taggarse A, Burkhart HM, et al. Robotic mitral valve repair for simple and complex degenerative disease: midterm clinical and echocardiographic quality outcomes. Circulation 2015;132:1961-8.

63. Smith CR, Leon MB, Mack MJ, et al. Transcatheter versus surgical aortic-valve replacement in high-risk patients. $\mathrm{N}$ Engl J Med 2011;364:2187-98.

64. Adams DH, Popma JJ, Reardon MJ, et al. Transcatheter aortic-valve replacement with a self-expanding prosthesis. N Engl J Med 2014;370:1790-8.

65. Thyregod HG, Steinbrüchel DA, Ihlemann N, et al. Transcatheter versus surgical aortic valve replacement in patients with severe aortic valve stenosis: 1 -year results from the all-comers NOTION randomized clinical trial. J Am Coll Cardiol 2015;65:2184-94.

66. Leon MB, Smith CR, Mack MJ, et al. Transcatheter or surgical aortic-valve replacement in intermediate-risk patients. N Engl J Med 2016;374:1609-20.

67. Siontis GC, Praz F, Pilgrim T, et al. Transcatheter aortic valve implantation vs. surgical aortic valve replacement for treatment of severe aortic stenosis: a meta-analysis of randomized trials. Eur Heart J 2016;37:3503-12. 